\title{
16
}

\section{Partially Ordered Sets - A Computerized Tool to Compare Environmental Databases}

\author{
Brüggemann, R., Voigt, $K$. \\ GSF - Forschungszentrum für Umwelt- und Gesundheit \\ Projektgruppe Umweltgefährdungspotentiale von Chemikalien \\ (PUC), \\ Neuherberg, 85758 Oberschleißheim, Germany \\ Telephone: ++49-89-3187-4030, Fax.: ++49-89-3187-3369 \\ e-mail:VOIGT@wiz.uni-kassel.de
}

Halfon, E.

National Water Research Institute

Canada Centre for Inland Waters

Burlington, Ontario, Canada L7R 4A6

Telephone: ++1-905-336-4917, Fax.: ++1-905-336-4972

e-mail:u044@cs.cciw.ca

\begin{abstract}
A set of objects can be analyzed by several tools. When ranking is a concern, then lattice theory and its graphical representation (Hasse diagrams) are useful. This paper introduces a new approach to analyze Hasse diagrams with respect to ranking in the environmental field. Simple set theoretical and lattice theoretical concepts such as successor sets, their cardinalities and the intersection of these sets have been used to compute a new matrix $\mathbf{D}$ that identifies the relation among objects. As an example we analyze a published data set of 22 objects (online databases of chemical and environmental relevance). These objects are scored using a six number scoring system with five environmental and chemical attributes. The online databases Toxline, Environmental Research Projects and Chemical Compliance Monitor turn out to be the "best" that is to say maximal elements in this scoring approach and within the concepts of lattice theory. Furthermore the importance which attribute contributes for the ranking procedure is shown. For this purpose we have introduced another new matrix, $\mathbf{W}$, which quantifies the dissimilarity of different Hasse diagrams and a sensitivity measure $s(E)$ that analyzes the importance of criteria, by which the environmental databases are
\end{abstract}


characterized. A crude estimation of the upper bound of $\mathrm{s}(E)$ is given. It can be shown thatthe attributes: "information parameter for environmental chemicals" (IP) and "number of chemicals" (NU) are relatively important and the attribute "Use of chemicals" (US) is relatively unimportant for the ranking of the chosen 22 environmental databases.

\section{Keywords}

Environmental databases, Hasse diagrams, ranking, sensitivity analysis.

\section{INTRODUCTION}

The basis of the Hasse diagram technique is the assumption that a ranking can be performed while avoiding the use of an ordering index (Halfon, 1986). In our application, Hasse diagrams present information not only on ranking but, most of all, they show whether the criteria, characterizing the objects, lead to ambiguities in the ranking procedure: For example, an object might be ranked higher according to one criterion but lower according to another. These two objects are not ordered because their data are "contradictory" to each other. This ambiguity -which is important to know for further applications- is not evident when we use an index for ranking, but it is immediately evident in a Hasse diagram. Hasse diagrams are extremely useful if several criteria are given to decide which objects are priority objects. In our approach the objects are environmental databases. The criteria are environmental and chemical ones and will be given later. The scoring is carried out according to a six number scoring system. Here we can not only identify the priority databases but furthermore it can be shown that these databases have different qualities.

In this paper we investigate a method to extract information from Hasse diagrams since the casual user of the ranking method of Halfon (1986) might become confused by the complexity given of the graphical information in a Hasse diagram and might not be able to use it. Furthermore, we study the influence of the choice of criteria to rank a set of objects (precise definitions follow later). The ranking of a set of objects does not only depend on the numerical values, but even more on the choice of criteria.

The results of this analysis are two matrices, $\mathbf{D}$ and $\mathbf{W}$, that identify the main features of the structure of Hasse diagrams and quantify the influence of criteria on the ranking.

\section{HASSE DIAGRAM TECHNIQUE APPLIED IN ENVIRONMENTAL PROTECTION}

The Hasse diagram technique has been already applied in several fields to rank objects of environmental relevance (see for example Brüggemann, 1995 b, c and Voigt, 1994). 


\section{THEORY OF HASSE DIAGRAM TECHNIQUE}

Davey and Priestley (1990) present useful background information on graphs, sets, partially ordered sets (posets) and Hasse diagrams. A few theoretical explanations will be given in the following paragraphs for a better understanding of the ranking procedure.

\subsection{Hasse diagram}

Hasse diagrams visualize the order relations within posets. Two objects $\mathrm{x}, \mathrm{y}$ of a poset are ordered if all scores of $\mathrm{x}$ are less or equal than those of $\mathrm{y}$. Hasse diagrams are oriented graphs (digraphs). A digraph consists of a set $E$ of objects drawn as small circles in Hasse diagrams. In our applications the circles near the top of the page (of the Hasse diagram) indicate objects that are the "best" objects according to the criteria used to rank them: These objects have no predecessors (they are not "covered" by other objects) and are called maximal elements, abbreviated also to "maximals."
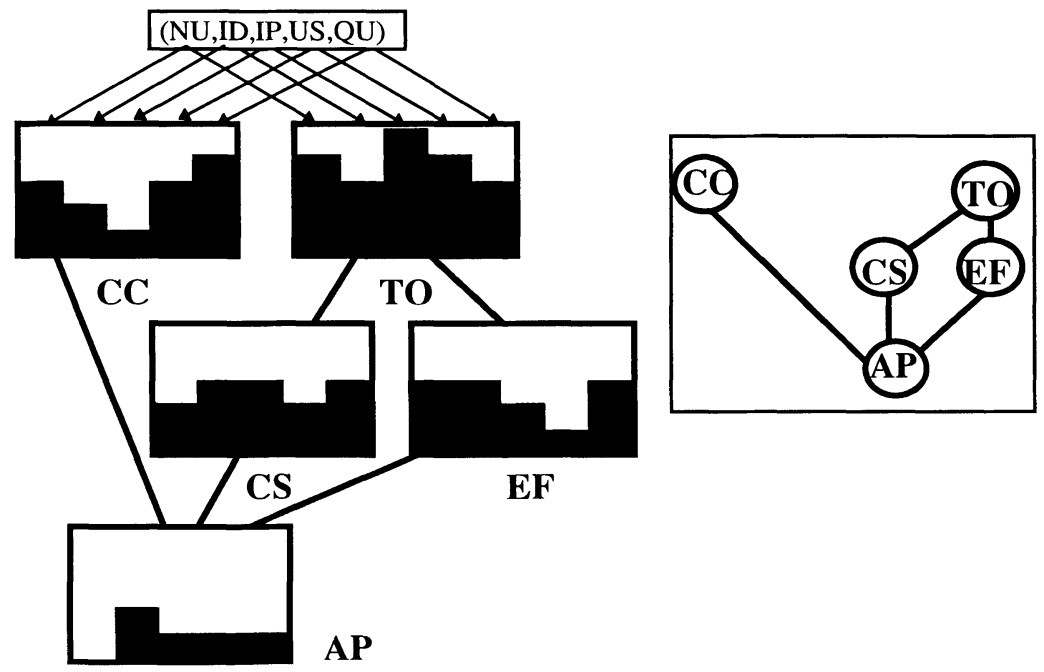

Figure 1 Two representations of a simplified Hasse diagram.

A line in the Hasse diagram indicates that the two objects connected by that line are "comparable" with each other, lack of sequences of connecting lines indicates that there are contradictions (a complete explanation with examples may be found in Halfon, 1986). A simple Hasse diagram will exemplify the above given statements; it is a part of the later discussed full diagram.

In Figure 1 the left diagram is an extended form of a Hasse diagram, where the scores are shown in the form of bar diagrams. We use the same scores 0 to 5 as in the full diagram (see below). The assignment of the attributes NU, ID, IP, US, QU (see chapter 5.1) to the bars is 
indicated by the arrows in the upper part of the left side diagram. The objects (environmental databases) are

$\mathrm{CC}=$ Chemical Compliance Monitor

$\mathrm{TO}=$ Toxicology Information Online

$\mathrm{CS}=$ Chemical Safety Newsbase

$\mathrm{EF}=$ Environmental Fate Database

$\mathrm{AP}=$ Air Pollution Technical Information Center

For example the two databases AP and TO are ordered, i.e. AP $\leq$ TO.

On the right side the usual shorthand representation of Hasse diagrams is shown, the assignments of the scores to the objects are usually given in a table.

\subsection{Definitions of basic terms}

Criteria include both quantitative and qualitative properties.

An attribute is a quantitative, measurable, criterion. Generally, we denote these attributes as $\# 1, \# 2, \ldots, \#$. It is convenient to denote the full attribute set as $A$. We call the full set of attributes an information basis (IB) of the ranking. A family of $\mathrm{p}:=2^{\mathrm{n}}-1$ attribute sets will be considered in our analysis, namely the power set of $A$ without the empty set. Each subset of attributes is denoted by $A_{\mathrm{i}}$, with $A_{\mathrm{i}} \subset A$ and will be used to perform a sensitivity analysis (see later).

Data are the numerical values corresponding to each criterion by which a given object is characterized.

An object is the item of interest. The set of $\mathrm{m}$ objects is called $E$. Each object is characterized by pairs, triples, quadrupels or generally by a tuple of data. Objects are ranked graphically by Hasse diagrams, as explained in 3.1.

Equivalent objects in Hasse diagrams: Different objects that have the same data with respect to a given set of attributes. They are elements of an equivalence class with the equivalence relation "equality of the characterizing tuples."

A case is a shorthand notation for an analysis by Hasse diagrams of $\mathrm{m}$ objects and with a defined attribute set $A_{\mathrm{i}}$. Thus, a given set of attributes induces a partially ordered set, which is visualized by a Hasse diagram.

\subsection{Definition of the terms "key elements" and "succcessor sets"}

Substructures within a Hasse diagram, i.e., relations among objects as well as the importance of criteria in ranking are investigated with the help of key elements and successor sets. Any object of the poset can be chosen as a starting point to begin the analysis. We call this object, a "key element". For convenience, all chosen key elements are considered to be elements of a set $K$, a subset of $E$.

In a Hasse diagram objects are connected by lines. Analysis of the successors of a key element implies a search of all objects located lower than the key element and connected to it by a path, being a sequence of connecting lines or equivalent to that of the key element. The set of all successors of a key element $\mathrm{k}$ is denoted as $G(\mathrm{k})$. The properties of the 
successor set $G(\mathrm{k})$ and its relation with successor sets of other key elements are first used to analyze the structure of a Hasse diagram, later they will be used to perform the sensitivity analysis. We write the cardinality of the successor set $G(\mathrm{k})$ as card $G(\mathrm{k})$. The cardinality of a set is its number of objects.

\subsection{Relations between elements and posets}

In order to investigate the global structure of the relations between any two elements of the posets, we perform this analysis mathematically. This structural relation might be hidden within the geometrical representation of the Hasse diagram. Thus, we introduce the symmetrical matrix $\mathbf{D}$, the entries of which are calculated from the cardinalities of all intersections of pairs of successor sets:

$$
\mathrm{D}_{\mathrm{ij}}:=\operatorname{card}[G(\mathrm{i}) \cap G(\mathrm{j})]^{\circ}
$$

The matrix $\mathbf{D}$ may be the basis to define several quantities describing the relation between objects within the posets quantitatively and is related to the sensivity analysis. Here we use entries of the matrix $\mathbf{D}$ to look for:

- the representativity of any key element " $\mathrm{i}$ " which is - if exactly one poset is considered given by $\mathrm{D}_{\mathrm{ii}}$ and

- the number of common elements between any two key elements $\mathrm{i}$ and $\mathrm{j}$.

Both numbers give the wanted rough insight into the Hasse diagram. We call this search for relations between objects an "unfolding" of the structure of the Hasse diagram.

Especially $D_{i j}(i \neq j)$ is of interest:

If $\mathrm{D}_{\mathrm{ij}}$ has a high value then the two key elements have many successor elements in common: they are comparable to many objects with respect to all attributes.

If $\mathrm{D}_{\mathrm{ij}}$ has a small value then the two key elements may be taken as representatives for two quite different sets of objects.

\section{HASSE PROGRAM}

The theory of the Hasse diagram technique is transfered to a DOS-Program Hasse, written in C. A WINDOWS-Version is anticipated.

The program is able to perform the following facilities:

- file organisation

- text-editing-functions

- data manipulation

- general characteristics of the posets

- visualization of posets by the corresponding Hasse diagram

- calculation of matrix D which entries may be used to study relations between key elements. We avoid the constructions of explicitly measures of similarity because further studies should be performed to establish an appropriate definition.

- setting-up and manipulation of diagrams

- performing of sensitivity analysis (standard and expert mode). 
The program is driven using pull-down-menus and comprises a help function which could still be more extensive. The Hasse program is in the test period at the moment but will be put on the market in the near future (Brüggemann 1995a).

\section{RANKING OF ENVIRONMENTAL DATABASES APPLYING HASSE DIAGRAM TECHNIQUE}

\subsection{Ranking of 22 Online Databases}

Since 1993 we develop priority setting concepts for data-sources, using the scientific background of lattice theory and its evaluation by Hasse diagrams.

Voigt and Brüggemann have scored 22 environmental online databases using the following five environmental and chemical-relevant evaluation criteria (Voigt, 1994):

- number of chemicals (NU)

- identification parameters (ID)

- information parameters for environmental chemicals (IP)

- use of chemical substances (US)

- quality of database (QU) (Voigt, 1994).

The scoring is carried out according to a six number scoring system; a high value means a high quality whereas a low value has the meaning of a low quality with respect to the criterion used.

In Figure 2 the Hasse diagram of the poset of 22 online databases evaluated by the information basis $\{\mathrm{NU}, \mathrm{ID}, \mathrm{IP}, \mathrm{US}, \mathrm{QU}\}$ is shown. The interpretation of the diagram might be difficult because of the many circles and lines. The concept of matrix $\mathbf{D}$ to this Hasse diagram is applied not only to explain its use but also to facilitate the interpretation of Figure 2. In this diagram three maximals exist. These are the databases UF (UmweltforschungsDatenbank, Environmental Projects Database), CC (Chemical Compliance Monitor) and TO (Toxicology Information Online). These databases (objects) are now defined to be the key elements. In Table 1 we list the elements of the successor sets.

Table 1 Successor sets of the databases TO, CC and UF

\begin{tabular}{|l|c|c|}
\hline \multicolumn{1}{|c|}{ TO } & $C C$ & $U F$ \\
\hline AP, AQ, AR, AS, BA & AP & AR \\
CB, CS, EB, EF, EN & AR & BA \\
EV, GE, NE, OC, PO & BA & EB \\
SC, UL, WA, WR & EN & EN \\
\hline 19 & NE & SC \\
\hline
\end{tabular}

The cardinalities of the three successor sets are:

card $G(\mathrm{TO})=19$

card $G(\mathrm{CC})=5$

card $G(\mathrm{UF})=5$ 


\subsubsection{Hasse Diagram for 22 Online Databases}

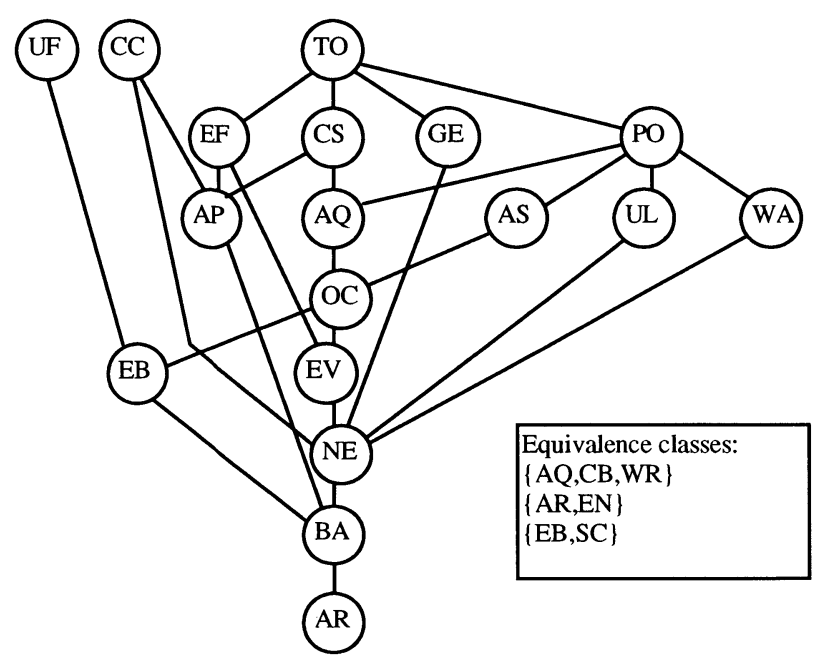

Figure 2 Hasse diagram for 22 Online Environmental Databases.

\subsubsection{Example to use the entries of matrix $D$}

The part of the intersection matrix $\mathbf{D}$, referring to the three objects (i.e. $K=\{T O, C C, U F\}$ ) of interest is:

$\begin{array}{cccccc} & & & 1 & 2 & 3 \\ & & & \text { TO } & \text { CC } & \text { UF } \\ & 1 & \text { TO } & 19 & 5 & 5 \\ & 2 & \text { CC } & 5 & 5 & 3 \\ & 3 & \text { UF } & 5 & 3 & 5\end{array}$

The difference of the cardinalities of the two successor sets of TO and CC is $14\left(D_{11}-D_{22}\right)$. TO has more successors (19) than CC (5). Therefore we conclude that even if TO and CC are ranked as maximals, TO represents more other databases which can be compared with this maximal than $\mathrm{CC}$ which has only 5 successors.

The cardinality of the intersection $\left(D_{12}=D_{21}\right)$ of these two successor sets is 5 , the same as the cardinality $\left(\mathrm{D}_{22}\right)$ of $G(\mathrm{CC})$ which is 5 . We conclude that even if the attributes of TO and $\mathrm{CC}$ make these two databases incomparable to one another, those databases represented by CC, namely AP, NE, BA, AR, EN are also comparable to that of TO. Similar argumentation can be found for TO and UF. 


\subsection{Sensivity Analysis of Ranking with Respect to its Attributes}

\subsubsection{Theory of Sensitivity Analysis}

The ranking of the objects is sensitive to the set of attributes. To quantify the importance of an attribute on ranking the basic idea is to compare Hasse diagrams induced by different attribute sets with each other. In order to do this, the concept of successor sets generated by a key element is the starting point again. The notation of successor set must be expanded to include all the actual combinations of attributes. Within the generalization of having a family of attribute sets the successor set depends not only on the key element but also on the choice of attributes. Therefore the following notation $G(\mathrm{k}, A)$ or $G\left(\mathrm{k}, A_{\mathrm{i}}\right)$ is used, where $G$ is the successor set, $\mathrm{k}$ denotes some arbitrary chosen key element, $A$ is the full set of attributes and $A_{\mathrm{i}}$ is a subset of attributes, $A_{\mathrm{i}} \subset A$.

The influence of each attribute can be quantified by counting the elements of the symmetrized difference between two successor sets, their so-called Hamming distance as follows:

$\mathrm{W}\left(\mathrm{k}, A_{\mathrm{i}}, A_{\mathrm{j}}\right):=\operatorname{card}\left\{\left[G\left(\mathrm{k}, A_{\mathrm{i}}\right) \backslash G\left(\mathrm{k}, A_{\mathrm{j}}\right)\right] \cup\left[G\left(\mathrm{k}, A_{\mathrm{j}}\right) \backslash G\left(\mathrm{k}, A_{\mathrm{i}}\right)\right]\right\}$

For a given key-element, two Hasse diagrams (given by two arbitrary sets of attributes) are more dissimilar, the more the successor sets $G\left(\mathrm{k}, A_{\mathrm{i}}\right)$ and $G\left(\mathrm{k}, A_{\mathrm{j}}\right)$ differ.

To simplify notation, we write $\mathrm{W}(\mathrm{k}, \mathrm{i}, \mathrm{j})$ for $\mathrm{W}\left(\mathrm{k}, A_{\mathrm{i}}, A_{\mathrm{j}}\right)$. We also note that each

$$
\mathrm{W}(\mathrm{k}, \mathrm{i}, \mathrm{j}) \geq 0 \text { and } \mathrm{W}(\mathrm{k}, \mathrm{i}, \mathrm{j})=\mathrm{W}(\mathrm{k}, \mathrm{j}, \mathrm{i}) \text {. }
$$

The matrix itself is denoted by $\mathbf{W}(\mathrm{k})$. Thus we have:

$$
\begin{aligned}
& \mathrm{W}(\mathrm{k}, 1,1), \mathrm{W}(\mathrm{k}, 1,2), \ldots ., \mathrm{W}(\mathrm{k}, 1, \mathrm{p}) \\
& \mathrm{W}(\mathrm{k}, 2,1), \mathrm{W}(\mathrm{k}, 2,2), \ldots ., \mathrm{W}(\mathrm{k}, 2, \mathrm{p})
\end{aligned}
$$

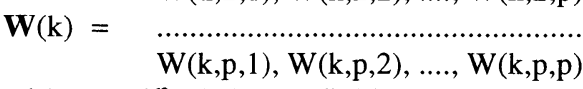

with $p=2^{n}-1$ (see definition of attribute). This matrix $\mathbf{W}$ is the key for the sensitivity analysis of ranking, each entry of $\mathbf{W}$ is the cardinality of the symmetrized difference (Equation 2) of two successor sets which are constructed from a given key element $\mathrm{k}$ and the two Hasse diagrams induced by two attribute subsets. This large but symmetrical matrix does not need to be analyzed in its entirety all the time because we are only interested in some few attribute sets (the standard mode of sensitivity analysis which is explained here). The sensitivity analysis can be performed by the following steps:

1) As we are only interested in comparisons of the full attribute set $A$ with subsets $A_{\mathrm{i}}$, only one row of the matrix $\mathbf{W}$ is of interest. We can choose the first one without loss of generalization, thus we are left with $\mathrm{W}(\mathrm{k}, 1,1), \mathrm{W}(\mathrm{k}, 1,2), \ldots ., \mathrm{W}(\mathrm{k}, 1, \mathrm{p})$, where the index 1 denotes the full attribute set $A$.

2) To see the influence of attributes on a Hasse diagram we compare the Hasse diagrams induced by $A$ with those induced by the attribute sets with only $\mathrm{n}-1$ attributes. Therefore the effect of dropping exactly one attribute on the ranking is given by the remaining $\mathrm{n}$ entries of the first row, $\mathrm{W}(\mathrm{k}, 1,2), \ldots ., \mathrm{W}(\mathrm{k}, 1, \mathrm{n}+1)$.

3) The remaining $\mathrm{n}$ matrix elements of the first row $\mathrm{W}\left(\mathrm{k}, A, A_{1}\right), \ldots ., \mathrm{W}\left(\mathrm{k}, A, A_{\mathrm{n}}\right)$ are put together to form a "sensitivity tuple" of the key element $\mathrm{k}$ :

$\sigma(\mathrm{k}):=(\mathrm{s}(\mathrm{k}, 1), \mathrm{s}(\mathrm{k}, 2), \ldots, \mathrm{s}(\mathrm{k}, \mathrm{n}))=\left[\mathrm{W}\left(\mathrm{k}, A, A_{1}\right), \ldots \mathrm{W}\left(\mathrm{k}, A, A_{\mathrm{n}}\right)\right]$.

Note that the enumerations of the subset $A_{\mathrm{i}}$ are thought of as follows: 


$$
\begin{aligned}
& A_{1}=\{\# 2, \ldots, \# \mathrm{n}\} \\
& A_{\mathrm{i}}=\{\# 1, \ldots, \# \mathrm{i}-1, \# \mathrm{i}+1, \ldots, \# \mathrm{n}\} \\
& A_{\mathrm{n}}=\{\# 1, \ldots, \# \mathrm{n}-1\}
\end{aligned}
$$

4) The larger the entries $\mathrm{s}(\mathrm{k}, \mathrm{i})$ the larger is the symmetrized difference between $G(\mathrm{k}, A)$ and $G\left(\mathrm{k}, A_{\mathrm{i}}\right)$ and correspondingly the larger the influence of attribute \#i on the position of key element $\mathrm{k}$ within the Hasse diagram under $A$ compared with that under $A_{\mathrm{i}}$.

5) The matrix $\mathrm{W}(\mathrm{k})$ depends on the selection of the key element $\mathrm{k}$. If however, more objects are to be analyzed we generalize as follows:

$$
\begin{aligned}
& \mathrm{W}(K, \mathrm{i}, \mathrm{j})=\sum \mathrm{W}(\mathrm{k}, \mathrm{i}, \mathrm{j}) \\
& \mathrm{k} \in K \subseteq E
\end{aligned}
$$

where $K$ is any set of key elements, in a shorter notation $\mathbf{W}(K)=\sum \mathbf{W}(\mathrm{k})$.

6) All objects are selected as key elements. Therefore instead of $\mathrm{W}(\mathrm{k}), \mathrm{W}(E)$ is to be investigated. $\mathrm{W}(E)$ is the total matrix of the set $E$. We note that a crude upper limit of $\mathrm{W}(E, \mathrm{i}, \mathrm{j})$ can be found simply by comparing a poset of $\mathrm{m}$ solely non-comparable elements with a poset where all $\mathrm{m}$ elements are equivalent to each other:

$$
0 \leq \mathrm{W}(E, \mathrm{i}, \mathrm{j}) \leq \mathrm{m}(\mathrm{m}-1)
$$

7) $\quad \mathbf{W}(E)$ will be used as a measure of sensitivity. Accordingly we suggest to quantify the sensitivity by:

$$
\mathrm{s}(E, \mathrm{i})=\mathrm{W}\left(E, A, A_{\mathrm{i}}\right) \quad 1 \leq \mathrm{i} \leq \mathrm{n}
$$

with the enumeration scheme of (5). According to (7), $\mathrm{s}(E, \mathrm{i})$ has values between 0 and $\mathrm{m}(\mathrm{m}-1)$.

The tedious calculation of $\mathrm{W}$ ( $E, A, A_{\mathrm{i}}$ ) by the defining equation (2) is not necessary. It can be shown that the following equation is valid (Brüggemann and Halfon: publication in preparation).

$$
\mathrm{s}(E, \mathrm{i})=\sum_{\mathrm{j}}\left(\mathrm{D}_{\mathrm{jj}}\left(A_{\mathrm{i}}\right)-\mathrm{D}_{\mathrm{jj}}(A)\right)
$$

where $\mathrm{D}\left(A_{\mathrm{i}}\right)$ and $\mathrm{D}(A)$, denotes the matrices $\mathbf{D}$ under attribute $A_{\mathrm{i}}$ and $A$.

\subsubsection{Application on the Example Hasse Diagram}

The entries of matrix $\mathbf{W}(E)$ corresponding to the comparisons of the successor sets induced by the full attribute sets and of those induced by $n$ other attribute sets where one attribute is omitted, are shown in Table 2. If we compare all attibutes used here, the attribute US (Use of chemicals) has a low, whereas the attributes IP (information parameter for environmental chemicals) and NU (number of chemicals) have a high influence on the ranking by Hasse diagrams.

Table 2 Results of the sensitivity analysis (standard mode)

\begin{tabular}{|llll|}
\hline $\begin{array}{l}\text { full attribute set } \\
\text { compared with case No. }\end{array}$ & attribute sets & omitted attribute & $\mathrm{s}(E, \mathbf{i})$ \\
\hline 2 & $A, A_{1}$ & QU & 20 \\
3 & $A, A_{2}$ & US & 11 \\
4 & $A, A_{3}$ & IP & 34 \\
5 & $A, A_{4}$ & ID & 16 \\
6 & $A, A_{s}$ & NU & 34 \\
\hline
\end{tabular}


Such finding is of importance for the practical use of the ranking procedure. In our example we are looking for priority environmental databases. Their identification depends on the actual criteria used. If NU is omitted, the analysis tell us that there are more changes in the diagram then by leaving out the criterion US. If IP is left out, the whole diagram changes, however the number of priority databases remains. Beyond this, it is of interest to discuss the attributes themselves and their role on the ranking procedure. A less important attribute may result from a positive correlation with another attribute.

\section{FURTHER DEVELOPMENTS}

The concept of partially ordered sets and their visualization by Hasse diagrams turns out to be very useful in many applications, where evaluative considerations are important. This applies to a large extent to environmental protection subjects. A recent - but brief - overview may be found in Brüggemann 1995 b, c. Considerable work is done to use the concept of posets as a modeling tool (Brüggemann, $1995 \mathrm{~b}$ ) and to derive from posets elements of artifical elements (Wille 1987). At least the underlying mathematical theory of lattices allows to deduce a system of implications (rules). However the applications of this concept for environmental purposes is on its very beginning, but is going to be intensified by our working groups in Germany and Canada in the near future.

\section{REFERENCES}

Brüggemann, R., Halfon, E. (1995a) Theoretical Base of the Program Hasse, GSF Forschungszentrum für Umwelt und Gesundheit, Neuherberg, in print.

Brüggemann R., Zelles L., Bai Q.Y., Hartmann A. (1995b) Use of Hasse Techniques for Evaluation of Phospholipid Fatty Acids Distributtion as Biomarkers in Selected Soils, Chemosphere, accepted.

Brüggemann R., Schwaiger J., Negele R.D. (1995c) Applying Hasse Diagram Technique for the Evaluation of Toxicological Fish Tests, Chemosphere, in press.

Davey, B. A. and Priestley, H.A. (1990) Introduction to Lattices and Order. Cambridge University Press, Cambridge.

Halfon, E. and Reggiani, M.G., (1986) On ranking chemicals for environmental hazard. Environ. Science Tech., 23, 1173-1179.

Voigt K., Brüggemann R. (1994) Umwelt-relevante Online Datenbanken im Vergleich, in Gesellschaft Deutscher Chemiker, Fachgruppe Chemie-Information-Computer (CIC), Mitteilungsblatt Nr. 29, GDCH, Frankfurt am Main, Mai 1994. 33-57.

Wille R. (1987) Bedeutung von Begriffsverbänden, in Ganter B., Wille R., Wolf K.E., Beiträge zur Begriffsanalyse 161-211, BI Wissenschaftsverlag, Mannheim. 


\section{Brüggemann, Rainer}

Rainer Brüggemann studied chemistry in Munich and made his Ph.D. 1977 in Theoretical Chemistry at the University of Munich. From 1980 to 1983 he worked at the Technical University of Berlin on the subject of safety studies for nuclear waste disposal sites. In 1984, he moved to the GSF National Research Center for Environment and Health in Neuherberg. There he worked on risk analysis and ecological modelling and QSAR in the field of chemicals. In 1992 he became head of the project group on environmental hazard assessment for chemicals. He published more than 100 articles, reports and books on chemistry and several environmental issues.

\section{Voigt, Kristina}

Kristina Voigt graduated in food chemistry at the Technical University in Berlin in 1979. Then she worked at the German Environmental Protection Agency (UBA) in Berlin. In 1983 she moved to the GSF National Research Center for Environment and Health in Munich. There her main task is testing and evaluation chemical-relevant and environmental-relevant data-sources. Since 1988 she has been head of the research project "Information System for Environmental Chemicals". She has published extensively in books, reports, proceedings and journals. She holds training courses, seminars and lectures. She is a member of the editorial board of Online \& CD-ROM Review. 